\title{
Verifying Sierpiński and Riesel Numbers in ACL2
}

\author{
John R. Cowles Ruben Gamboa \\ Department of Computer Science \\ University of Wyoming \\ Laramie, Wyoming, USA \\ cowles@cs . uwyo.edu \\ ruben@cs . uwyo.edu
}

A Sierpiński number [4, page 420] and [1], is an odd positive integer, $k$, such that no positive integer in this infinite list is prime:

$$
k 2^{1}+1, k 2^{2}+1, k 2^{3}+1, \ldots, k 2^{n}+1, \ldots
$$

A Riesel number [1] is similar to a Sierpiński number, with -1 replacing +1 in the above infinite list. Such a number is an odd positive integer, $k$, so that no positive integer in this infinite list is prime:

$$
k 2^{1}-1, k 2^{2}-1, k 2^{3}-1, \ldots, k 2^{n}-1, \ldots
$$

A cover, for such a $k$, is a finite list of positive integers such that each integer, $j$, in the appropriate infinite list, has a factor, $d$, in the cover, with $1<d<j$.

Given a $k$ and its cover, ACL2 is used to systematically verify that each integer, in the appropriate infinite list, has a smaller factor in the cover.

\section{Introduction}

Sierpiński and Riesel numbers are not easy to find. To disqualify an odd positive integer as a Sierpiński number or a Riesel number, one need only locate a prime in the appropriate infinite list. With four exceptions, $k=47,103,143,197$, all of the first 100 odd positive integers, $1 \leq k \leq 199$, are disqualified as Sierpiński numbers by finding at least one prime in the first eight elements of the infinite list [3]:

$$
k 2^{1}+1, k 2^{2}+1, k 2^{3}+1, \ldots, k 2^{8}+1 .
$$

Both $k=103$ and $k=197$ are eliminated by finding a prime in the list no later than $k 2^{16}+1$ [3], leaving 47 and 143 as the only possible Sierpiński numbers less than 200. It turns out that $143 \cdot 2^{53}+1$ and $47 \cdot 2^{583}+1$ are prime [3], eliminating them. Thus, there are no Sierpiński numbers in the range $1 \leq k \leq$ 199. The situation is similar for Riesel numbers.

In 1960, W. Sierpiński [7] proved, for

$$
k=15511380746462593381,
$$

every member in the infinite list, given by (1), is divisible by one of the prime factors of the first six Fermat numbers. For nonnegative integer, $n$, the Fermat number, $F_{n}$, is given by

$$
F_{n}=2^{2^{n}}+1
$$

The first five Fermat numbers are prime and $F_{5}$ is the product of two primes:

$$
F_{0}=3, F_{1}=5, F_{2}=17, F_{3}=257, F_{4}=65537 \text {, }
$$

Hardin and Schmaltz (Eds): ACL2 2011

EPTCS 70, 2011, pp. 20-27 doi 10.4204/EPTCS.70.2 (c) J.R. Cowles \& R. Gamboa

This work is licensed under the Creative Commons Attribution-Noncommercial-No Derivative Works License. 


$$
F_{5}=4294967297=641 \cdot 6700417 .
$$

Thus (3 517257641655376700417 ) is a cover for $k=15511380746462593381$, showing it to be a Sierpiński number. Sierpiński's original proof is described in [8, page 374] and [2].

In 1962, J. Selfridge (unpublished) proved that 78557 is a Sierpiński number by showing that

$$
\text { (35 } 513193773)
$$

is a cover [6]. Later, in 1967, Selfridge and Sierpiński conjectured that 78557 is the smallest Sierpinski number [6]. The distributed computing project Seventeen or Bust [6] is devoted to proving this conjecture, disqualifying every $k<78557$, by finding an $n$ that makes $k \cdot 2^{n}+1$ prime. For example, $19249 \cdot 2^{13018586}+1$, a 3918990-digit prime, eliminated 19249 [9]. When this project started in 2002, all but 17 values of $k$ had already been disqualified. Currently six values of $k$ remain to be eliminated.

Earlier, in 1956, but less well known than Sierpiński's work, H. Riesel [5] showed 509203 is a Riesel number with cover (3 571317 241). It is possible for the same odd positive integer to be both a Sierpiński number and a Riesel number. An example [1] is $k=143665583045350793098657$.

\section{Covers Into ACL2 Proofs}

Given an odd positive integer, $k$, with a Sierpiński cover, $\mathscr{C}$, here is the process used to verify that $k$ is a Sierpiński number. There is a similar process for verfying Riesel numbers from their covers.

1. For each $d$ in $\mathscr{C}$, find positive integer $b_{d}$ and nonnegative integer $c_{d}$ so that for every nonnegative integer $i, d$ is a factor of $k \cdot 2^{b_{d} \cdot i+c_{d}}+1$.

In practice, every $d$ in $\mathscr{C}$ is an odd prime smaller than $k$.

(a) Search for positive integer $b$ such that $d$ is a factor of $2^{b}-1$. Since $d$ is an odd prime, it turns out that such a $b$ will always exis 1 among $1,2, \ldots, d-1$. Let $b_{d}$ be the first $\operatorname{such} b$.

(b) Search for nonnegative integer $c$ such that $d$ is a factor of $k \cdot 2^{c}+1$. If such a $c$ exists, then one exists among $0,1, \ldots, b_{d}-1$. Let $c_{d}$ be the firs $3^{3}$ such $c$, if it exists.

(c) Assuming $c_{d}$ exists, use induction on $i$, to prove that for every nonnegative integer $i, d$ is a factor of $k \cdot 2^{b_{d} \cdot i+c_{d}}+1$.

The base case, when $i=0$, follows from $1 \mathrm{~b}$ above.

The induction step, going from $i=j$ to $i=j+1$, follows from 1a above:

$$
k 2^{b_{d}(j+1)+c_{d}}+1=\left[k 2^{b_{d} j+c_{d}} \cdot\left(2^{b_{d}}-1\right)\right]+\left[k 2^{b_{d} j+c_{d}}+1\right]
$$

By $1 \mathrm{a}, d$ is a factor of the left summand of (2) and $d$ is a factor of the right summand by the induction hypothesis.

2. For each positive integer $n$, find $d$ in $\mathscr{C}$ and nonnegative integer $i$ so that $n=b_{d} \cdot i+c_{d}$. If such $d$ and $i$ exist, then, by $1 \mathrm{c}, d$ is a factor of $k \cdot 2^{b_{d} \cdot i+c_{d}}+1=k \cdot 2^{n}+1$.

To ensure that such $d$ and $i$ exist for every positive $n$, only a finite number of cases need be considered: Let $\ell$ be the least common multiple of all the $b_{d}$ 's found for the $d$ 's in $\mathscr{C}$. Check for each

$$
n \in\{0,1,2, \ldots, \ell-1\},
$$

\footnotetext{
${ }^{1}$ For the mathematically literate: The well-known Fermat's Little Theorem ensures the claimed existence.

${ }^{2}$ Thus, being mathematically precise, $b_{d}$ is just the order of 2 in the multiplicative group of the integers modulo $d$.

${ }^{3}$ If $d$ does not divide $k$, then $2^{c_{d}} \equiv-(1 / k) \quad(\bmod d)$, so $c_{d}$ is the discrete logarithm, base 2 , of $-(1 / k)$ in the integers modulo $d$.
} 
that there always is a $d$ in $\mathscr{C}$ that satisfies the equation

$$
\bmod \left(n, b_{d}\right)=c_{d}
$$

This process has not been formally verified in ACL2. For example, we don't bother to check that every member of $\mathscr{C}$ is an odd prime. Instead, for each individual $k$ and $\mathscr{C}$, ACL2 events are generated that would prove $k$ is a Sierpiński number, if all the events succeed. If some of the events fail, then, as usual when using ACL2, further study of the failure is required, in the hope of taking corrective action. The generation of these events is controlled by the macros verify-sierpinski and verify-riesel. These macros take three arguments: the name of a witness function that will find a factor for a given $k 2^{n} \pm 1$, the number $k$ that is a Sierpiński or Riesel number, and the cover $\mathscr{C}$ for $k$. The macros then generate the proof, following the plan outlined in this section.

For each $d$ in $\mathscr{C}, b_{d}$ and $c_{d}$ from $1 \mathrm{a}$ and $1 \mathrm{~b}$, are computed. They are needed to define the witness function and to state the theorems mentioned in $1 \mathrm{c}$, which are then proved. For example, the proof that 78557 is a Sierpiński number defines this witness function:

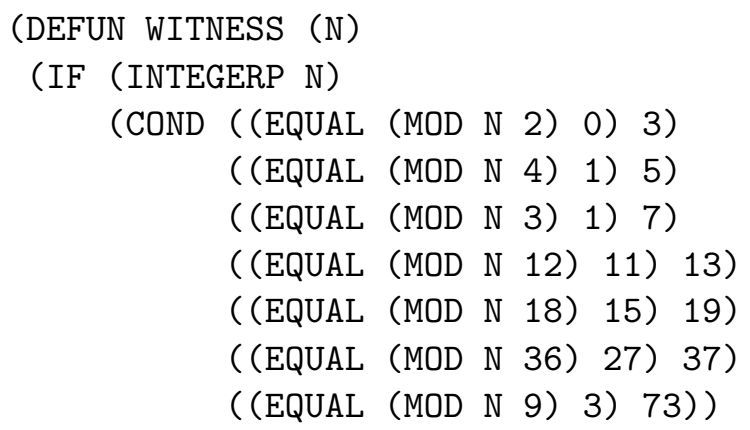

The rightmost numbers, in this definition, form the cover, the corresponding $b_{d}$ 's are the leftmost numbers, and the middle numbers are the $c_{d}$ 's. So $\mathscr{C}=\left(\begin{array}{l}3 \\ 5\end{array} 713193773\right), b_{73}=9$, and $c_{73}=3$.

The theorem, from 1c, for $d=73$ is

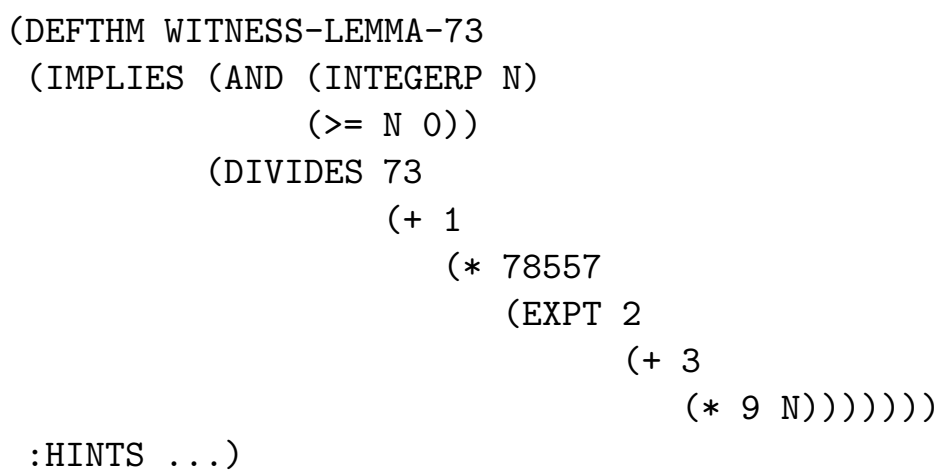

Four properties are proved about the witness function, establishing 78557 is a Sierpiński number:

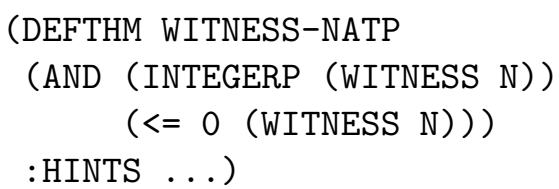




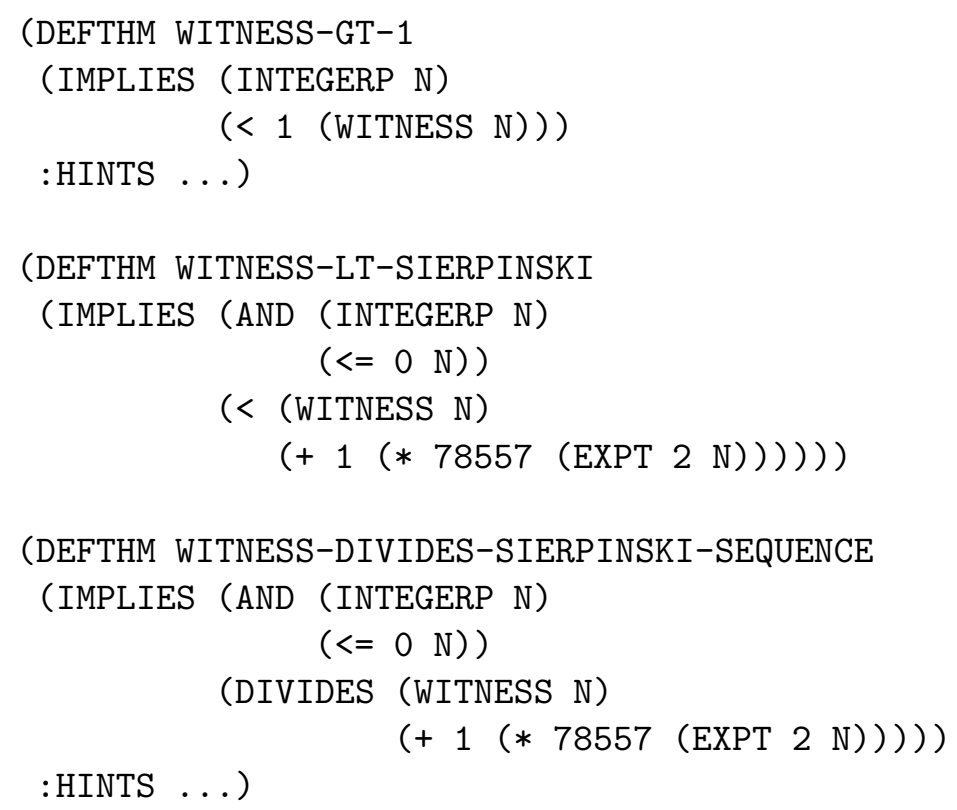

As suggested above in 2, these properties can be proved by showing every integer is "covered" by one of the cases given in the COND-expression used in the definition of the witness function.

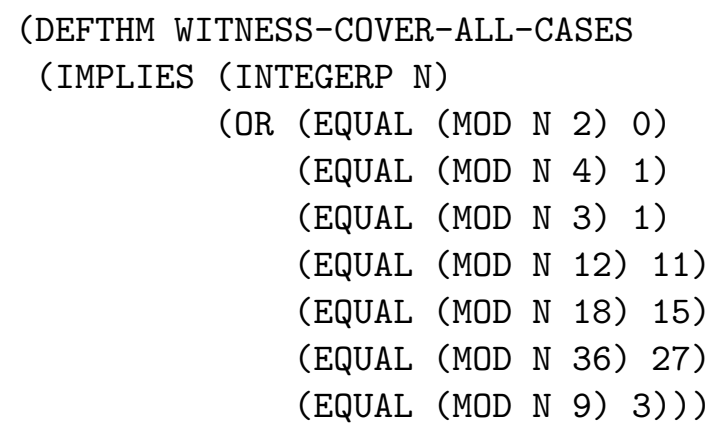

:RULE-CLASSES NIL :HINTS ...)

To prove this, we first demonstrate that these cases are exhaustive when $n$ is replaced by mod $(n, 36)$ (where 36 is the least common multiple of all the moduli above). This can be checked, essentially, by computation.

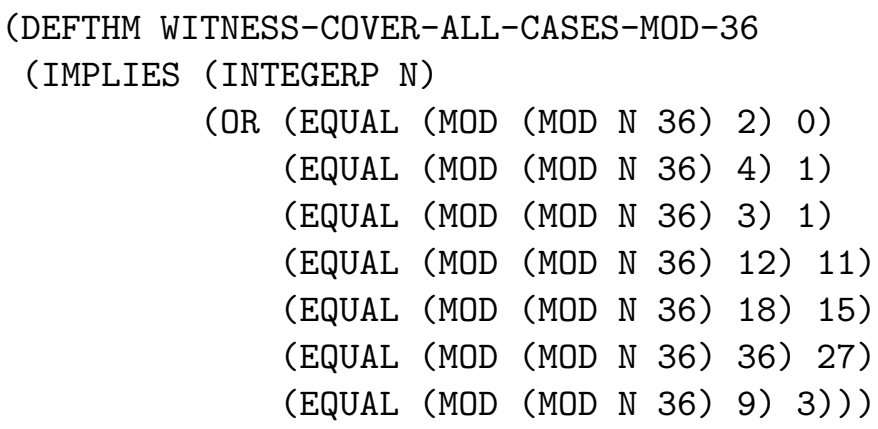


The actual modular equivalences that need to be proved depend on both the number 78557 and its cover. Although the theorem that is being proved is obviously true, there does not appear to be a way to prove it once and for all in ACL2, not even using encapsulate. Instead, a pair of theorems very much like the ones we have described needs to be proved from scratch for each different Sierpiński or Riesel number. As experienced ACL2 users, we are concerned that ACL2 will simply fail to prove this theorem for some combination of numbers and their covers. However, we have used these macros to generate the proof for each of the Sierpiński and Riesel numbers with covers listed in the appendix, and all of the proofs have gone through automatically. Note that the appendix essentially 4 contains all the Sierpiński and Riesel numbers known to us.

\section{Numbers Without Covers}

There are odd positive integers, shown to be Sierpiński (or Riesel) numbers, that have no known covers. ACL2 proofs have been constructed for these numbers.

For example [1], $k=4008735125781478102999926000625$ is a Sierpiński number, but no (complete) cover is known. For all positive integer, $n$, if $\bmod (n, 4) \neq 2$, then $k \cdot 2^{n}+1$ has a factor among the members of (3 1797241257673 ). To show $k$ is a Sierpiński number, a factor of $k \cdot 2^{n}+1$ must be found for all positive integer, $n$, such that $\bmod (n, 4)=2$. Such a factor is constructed using these facts:

- $k=44745755^{4}$ is a fourth power

- $4 x^{4}+1=\left(2 x^{2}+2 x+1\right) \cdot\left(2 x^{2}-2 x+1\right)$

Let $i=44745755$, so $k=i^{4}$. Then

$$
\begin{aligned}
k \cdot 2^{4 n+2}+1 & =2^{2}\left(i \cdot 2^{n}\right)^{4}+1 \\
& =4\left(i \cdot 2^{n}\right)^{4}+1 \\
& =\left[2\left(i \cdot 2^{n}\right)^{2}+2\left(i \cdot 2^{n}\right)+1\right] \cdot\left[2\left(i \cdot 2^{n}\right)^{2}-2\left(i \cdot 2^{n}\right)+1\right]
\end{aligned}
$$

The left factor of (3) algebraically reduces to show

$$
4004365181040050 \cdot 2^{2\lfloor n / 4\rfloor}+89491510 \cdot 2^{\lfloor n / 4\rfloor}+1
$$

is a factor of $k \cdot 2^{n}+1$, whenever $\bmod (n, 4)=2$.

A Riesel number, $k$, with no known cover, is given in Appendix A. In this example, $k=a^{2}$ is a square and

$$
\begin{aligned}
k \cdot 2^{2 n}-1 & =a^{2} \cdot 2^{2 n}-1 \\
& =\left(a 2^{n}\right)^{2}-1 \\
& =\left(a 2^{n}+1\right) \cdot\left(a 2^{n}-1\right)
\end{aligned}
$$

shows how to factor $k \cdot 2^{m}-1$ when $m$ is even and positive. A (partial) cover, listed in Appendix A, gives a constant factor for each $k \cdot 2^{m}-1$, when $m$ is odd and positive.

\footnotetext{
${ }^{4}$ Given a Sierpiński or Riesel number $k$ and its cover $\mathscr{C}$, infinitely many other examples can be constructed: Let $P$ be the product of the numbers in $\mathscr{C}$ and let $i$ be a positive integer. Then $k+2 \cdot i \cdot P$ is also a Sierpiński or Riesel number with the same cover.
} 


\section{Conclusions}

Given a Sierpiński or Riesel number, $k$, and its cover, we have described ACL2 macros that generate events verifying that each integer, in the appropriate infinite list, has a smaller factor in the cover.

For the few known Sierpiński or Riesel numbers with no known covers, hand-crafted ACL2 proofs have been constructed verifying that each integer, in the appropriate infinite list, has a smaller factor.

\section{References}

[1] Michael Filaseta, Carrie Finch \& Mark Kozek (2008): On Powers Associated with Sierpiński Numbers, Riesel Numbers and Polignac's Conjecture. Journal of Number Theory 128, pp. 1916-1940.

[2] Lenny Jones (2011): When Does Appending the Same Digit Repeatedly on the Right of a Positive Integer Generate a Sequence of Composite Integers? American Mathematical Monthly 118, pp. 153-160.

[3] Website: The On-Line Encyclopedia of Integer Sequences. http://oeis . org/A046067.

[4] Clifford Pickover (2009): The Math Book. Sterling Publishing, New York.

[5] Hans Riesel (1956): Några Stora Primtal (Swedish: Some Large Primes). Elementa 39, pp. 258-260.

[6] Website: Seventeen or Bust. www . seventeenorbust. com. A Distibuted Attack on the Sierpinski Problem.

[7] Waclaw Sierpiński (1960): Sur un Problème Concernant les Nombres $k \cdot 2^{n}+1$. Elem. Math. 15, pp. 73-74. Corrigendum, ibidem, 17:85, 1962.

[8] Waclaw Sierpiński (1987): Elementary Theory of Numbers. PWN-Polish Scientific Publishers and Elsevier Science Publishers, Warszawa and Amsterdam. Second English edition revised and enlarged by A. Schinzel.

[9] Website: The Sierpiński Problem: Definition and Status. www.prothsearch.net/sierp.html.

\section{A Sierpiński and Riesel Numbers}

Numbers, $k$, verified with ACL2.

Each $k$ with a cover $\mathscr{C}$ is either mentioned in the References or claimed at various websites. Numbers $k$ without known covers are from [1].

\section{Smallest known Sierpiński number}

$k=78557=17 \cdot 4621$, a product of two primes

$\mathscr{C}=\left(\begin{array}{ll}3 & 5713193773\end{array}\right)$

\section{Smallest known prime Sierpiński number}

$k=271129$

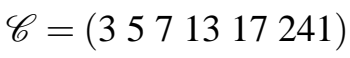




\section{More Sierpiński numbers}

\begin{tabular}{|c|c|}
\hline$k$ & $\mathscr{C}$ \\
\hline 271577 & 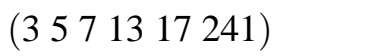 \\
\hline 322523 & (3 57133773109$)$ \\
\hline 327739 & (3 57131797257$)$ \\
\hline 482719 & $(3571317241)$ \\
\hline 575041 & $\left(\begin{array}{lllll}3 & 5 & 7 & 13 & 17 \\
241\end{array}\right)$ \\
\hline 603713 & $\left(\begin{array}{l}3 \\
5\end{array} 71317241\right)$ \\
\hline 903983 & $\left(\begin{array}{lllll}3 & 5 & 13 & 17 & 241\end{array}\right)$ \\
\hline 934909 & $\left(\begin{array}{l}3 \\
5\end{array} 7 \begin{array}{llll}13 & 19 & 73 & 109\end{array}\right)$ \\
\hline 965431 & $(3571317241)$ \\
\hline 1259779 & 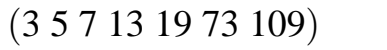 \\
\hline 1290677 & 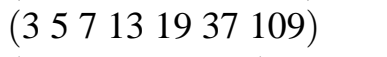 \\
\hline 1518781 & $(3571317241)$ \\
\hline 1624097 & $\left(\begin{array}{l}3 \\
5\end{array} 71317241\right)$ \\
\hline 1639459 & $\left(\begin{array}{l}3 \\
5\end{array} 71317241\right)$ \\
\hline 1777613 & 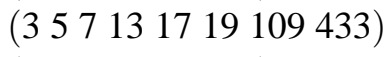 \\
\hline 2131043 & $\left(\begin{array}{l}3 \\
5\end{array} 71317241\right)$ \\
\hline
\end{tabular}

\section{Smallest Sierpiński number found by Sierpiński}

$k=15511380746462593381$

$\mathscr{C}=(3517257641655376700417)$

\section{Smallest known Riesel number}

$$
\begin{aligned}
& k=509203 \\
& \mathscr{C}=\left(\begin{array}{llll}
3 & 5713 & 17 & 241
\end{array}\right)
\end{aligned}
$$

\begin{tabular}{|c|c|}
\hline$k$ & $\mathscr{C}$ \\
\hline 762701 & $\left(\begin{array}{lllll}3 & 5 & 7 & 13 & 17 \\
241\end{array}\right)$ \\
\hline 777149 & $\left(\begin{array}{l}3 \\
5\end{array} 713193773\right)$ \\
\hline 790841 & $\left(\begin{array}{llllll}3 & 5 & 7 & 13 & 19 & 37\end{array}\right)$ \\
\hline 992077 & $\left(\begin{array}{l}35 \\
5\end{array} 71317241\right)$ \\
\hline
\end{tabular}

\section{More Riesel numbers}

\section{Numbers both Sierpiński and Riesel}

$\mathscr{C}_{R}$ indicates the Riesel number cover and $\mathscr{C}_{S}$ indicates the Sierpiński number cover.

$$
\begin{aligned}
& k=143665583045350793098657 \\
& \mathscr{C}_{R}=\left(\begin{array}{ll}
3 & 5131797241257
\end{array}\right) \\
& \mathscr{C}_{S}=\left(\begin{array}{ll}
3 & 1119313761731091513311321
\end{array}\right) \\
& k=47867742232066880047611079 \\
& \mathscr{C}_{R}=\left(\begin{array}{ll}
3 & 11193137416173109151331
\end{array}\right) \\
& \mathscr{C}_{S}=\left(\begin{array}{ll}
3 & 131797241257
\end{array}\right)
\end{aligned}
$$




$$
\begin{aligned}
& k=878503122374924101526292469 \\
& \mathscr{C}_{R}=\left(\begin{array}{ll}
371319377397109241257
\end{array}\right) \\
& \mathscr{C}_{S}=\left(\begin{array}{lll}
3511 & 1731416115133161681
\end{array}\right) \\
& \hline k=3872639446526560168555701047 \\
& \mathscr{C}_{R}=\left(\begin{array}{ll}
371319377397109241673
\end{array}\right) \\
& \mathscr{C}_{S}=\left(\begin{array}{ll}
35111731416115133161681
\end{array}\right) \\
& \hline k=623506356601958507977841221247 \\
& \mathscr{C}_{R}=\left(\begin{array}{lll}
3 & 71319377397109241673
\end{array}\right) \\
& \mathscr{C}_{S}=\left(\begin{array}{ll}
3 & 517257641655376700417
\end{array}\right)
\end{aligned}
$$

\section{Sierpiński numbers without cover}

$k=4008735125781478102999926000625=44745755^{4}$

(3 1797241257673$)$ is partial cover for $\bmod (n, 4) \neq 2$.

$4004365181040050 \cdot 2^{2\lfloor n / 4\rfloor}+89491510 \cdot 2^{\lfloor n / 4\rfloor}+1$

is a factor of $k \cdot 2^{n}+1$, whenever $\bmod (n, 4)=2$.

$k=734110615000775^{4}$

(3 17257641655376700417$)$ is partial cover for $\bmod (n, 4) \neq 2$.

$1077836790113632192906501201250 \cdot 2^{2\lfloor n / 4\rfloor}+1468221230001550 \cdot 2^{\lfloor n / 4\rfloor}+1$ is a factor of $k \cdot 2^{n}+1$, whenever $\bmod (n, 4)=2$.

\section{Riesel number without cover}

Let $a=3896845303873881175159314620808887046066972469809$ and let $k=a^{2}$.

The list

(7 173141719711312715124125728133764167313211444929191655376700417 ) is partial cover for odd positve $n$.

$a \cdot 2^{n / 2}+1$ is a factor of $k \cdot 2^{n}-1$, whenever $n$ is positive and even. 